\title{
Thermal Conductivity of Graphitic Carbon Nitride Nanotubes: A Molecular Dynamics Study
}

\author{
Hui Guo $\mathbb{D},{ }^{1}$ Chunqing Huo, ${ }^{2}$ Liang Yang $\mathbb{D}^{2},{ }^{2}$ and Shiwei Lin $\mathbb{D}^{2}$ \\ ${ }^{1}$ College of Science, Hainan University, Haikou 570228, China \\ ${ }^{2}$ School of Materials Science and Engineering, Hainan University, Haikou 570228, China \\ Correspondence should be addressed to Liang Yang; y15923@hainanu.edu.cn and Shiwei Lin; linsw@hainanu.edu.cn
}

Received 20 October 2021; Revised 22 November 2021; Accepted 26 November 2021; Published 15 December 2021

Academic Editor: Shuyuan Xiao

Copyright $($ C 2021 Hui Guo et al. This is an open access article distributed under the Creative Commons Attribution License, which permits unrestricted use, distribution, and reproduction in any medium, provided the original work is properly cited.

Graphitic carbon nitride $\left(\mathrm{g}-\mathrm{C}_{3} \mathrm{~N}_{4}\right)$ nanotubes are recently gaining increasing interest due to their extraordinary physicochemical properties. In the following, we report on simulations using a method of nonequilibrium molecular dynamics and focus on the thermal conductivity variation of g- $\mathrm{C}_{3} \mathrm{~N}_{4}$ nanotubes with respect to different temperatures, diameters, and chiral angles. In spite of the variation of diameters and chiral angles, the structure of nanotubes possesses high stability in the temperature range from $200 \mathrm{~K}$ to $600 \mathrm{~K}$. Although there is little change of the thermal conductivity per unit arc length for nanotubes with the same diameter at different temperatures, it decreases significantly with increasing diameters at the same temperature. The thermal conductivity at different chiral angles has little to do with how temperature changes. Simulation results show that the vibrational density of states of nanotubes distributed, respectively, at $\sim 11 \mathrm{THz}$ and $\sim 32 \mathrm{THz}$, indicating that heat in nanotubes is mostly carried by phonons with frequencies lower than $10 \mathrm{THz}$.

\section{Introduction}

Graphitic carbon nitride $\left(\mathrm{g}-\mathrm{C}_{3} \mathrm{~N}_{4}\right)$ is a two-dimensional semiconductor material with graphene-like single-layer structure [1]. It has attracted tremendous attention and has been widely applied in many fields because of the stable physicochemical and excellent electro-optical properties, such as the production of hydrogen from water photolysis [2], battery production [3], photocatalysis $[4,5]$, and electrochemistry [6] processes. Recently, one-dimensional nanotube structural g- $\mathrm{C}_{3} \mathrm{~N}_{4}$ analogous materials have been successfully prepared [7], and enormous progress has been made in both experimental research and theoretical explorations. On the one hand, g- $\mathrm{C}_{3} \mathrm{~N}_{4}$ nanotubes have been successfully prepared on porous $\mathrm{Al}_{2} \mathrm{O}_{3}$ anode plates via the sol-gel process. Bian et al. [8] further improved their hydrogen evolution ability by depositing $\mathrm{Pt}$ nanoparticles on the tube wall. g- $\mathrm{C}_{3} \mathrm{~N}_{4}$ nanotubes synthesized by the two-step method displayed 12 times higher photocatalytic efficiency than that of bulk materials when decomposing rhodamine B [9]. On the other hand, first-principle calculation was used to predict the structural stability and electronic properties of g- $\mathrm{C}_{3} \mathrm{~N}_{4}$ tubular composite materials, which indeed verified the stable existence of nanotube structural materials [10]. Pan et al. further discovered that electronic structures of g- $\mathrm{C}_{3} \mathrm{~N}_{4}$ nanotubes have adjustable band gaps and predicted that they could be used in the conversion of solar energy [11]. However, most of these research studies on g- $\mathrm{C}_{3} \mathrm{~N}_{4}$ nanotubes are focused on their photoelectric properties; very few discussed their thermodynamic properties, especially thermal conductivity. In many applications, $\mathrm{g}-\mathrm{C}_{3} \mathrm{~N}_{4}$ is often used to make composites, and thus, its thermal conductivity may significantly affect the nature of the composites and the catalysis process. The lack of cognition of the thermal conductivity of g- $\mathrm{C}_{3} \mathrm{~N}_{4}$ greatly prevents the in-depth understanding of $\mathrm{g}-\mathrm{C}_{3} \mathrm{~N}_{4}$ nanotubes and their applications.

Here, we have employed molecular dynamics (MD) simulation method to investigate the relation between the thermal conductivity of g- $\mathrm{C}_{3} \mathrm{~N}_{4}$ nanotubes and temperature, nanotube diameters, and chiral angles. It will be shown that the thermal mechanism of nanotubes is closely related with vibrational density of state, the nanotubes process high 
stability at a wide temperature range, thermal conductivity is closely related to diameters, and heat in nanotubes is mostly carried by phonons with certain frequencies.

\section{Computational Model and Method}

2.1. Computational Model. A model from Momma and Izumi is used in this study, as shown in Figure 1 [12].

At present, the most widely applied two-dimensional $\mathrm{CN}$ compound should be the $\mathrm{g}-\mathrm{C}_{3} \mathrm{~N}_{4}$ structure. Then, it is also applied here to construct nanotubes with the axial length of about $120 \mathrm{~nm}$. Based on the structural symmetry, the chiral angles at different chiral directions of the constructed nanotube cells were $m 1=0^{\circ}, m 2=6^{\circ}, m 3=20^{\circ}, m 4=30^{\circ}$, $m 5=40^{\circ}, m 6=54^{\circ}$, and $m 7=60^{\circ}$, respectively, as shown in Figure 1. In addition, different inner diameters $(0.71 \mathrm{~nm}$, $1.18 \mathrm{~nm}, 1.72 \mathrm{~nm}, 2.13 \mathrm{~nm}, 2.35 \mathrm{~nm}$, and $2.59 \mathrm{~nm})$ of nanotubes were also constructed at chiral angle $m 1=0^{\circ}$. The information of the constructed nanotubes and the computational parameters are shown in Table 1. The computation is implemented by Device Studio software.

2.2. Computational Method. Large-scale Atomic/Molecular Massively Parallel Simulator (LAMMPS) package, an opensource MD code, was applied in the MD simulation [13]. The accuracy of the MD simulation strongly depends on the classical force fields utilized to describe the interatomic forces. Potential proposed by Tersoff (Tersoff potential) [14] was used to calculate the interaction between carbon and nitrogen atoms. Nonequilibrium molecular dynamics method was employed for the computation of thermal conductivity. For a single nanotube, heat transferred directly from every hot end $(0.5 \mathrm{~nm}$ from the bottom end) to every cold end ( $0.5 \mathrm{~nm}$ away from the center of the length). Therefore, the thermal conductivity $(\kappa)$ can be directly obtained from the temperature gradient $(\Delta T)$ and the heat flux of the system $(J)$ through Fourier's formula [1]:

$$
\kappa=-\frac{J}{\Delta T}
$$

In order to keep the constant energy and momentum of the system, $0.2 \mathrm{eV}$ energy $(Q)$ was added into the hot end per ps, while the same energy $Q$ was subtracted from the cold end. Then, heat flow and a stable temperature gradient can be formed between the two ends in such a stable system. The heat flux can be determined by $J=Q / S$, where $S$ is the crosssectional area of the system. The temperature gradient is expressed as $\Delta T=\mathrm{d} T / \mathrm{d} L$, which is the derivative of the linear temperature change.

As shown in Figure 2, the temperature changes linearly in the axial direction in a stable system. Therefore, the thermal conductivity $(\kappa)$ can be calculated by

$$
\kappa=-\frac{\mathrm{Q} / \mathrm{S}}{\mathrm{d} T / \mathrm{d} L} \text {. }
$$

The phonon density of states is obtained by calculating the fast Fourier transform of the phonon spectrum from the velocity autocorrelation function (VACF) of the system and is responsible for the change of thermal conductivity with nanotube diameters and temperature. Here, VACF is calculated by the following formula:

$$
\operatorname{VACF}(t)=\frac{\left\langle\sum_{i=1}^{N} \vec{v}_{i}\left(t_{0}\right) \vec{v}_{i}\left(t_{0}+t\right)\right\rangle}{\left\langle\sum_{i=1}^{N} \vec{v}_{i}\left(t_{0}\right) \vec{v}_{i}\left(t_{0}\right)\right\rangle}
$$

where $N$ is the total atomic number of the system, $\vec{v}_{i}\left(t_{0}\right)$ is the velocity of the $i$ th atom at time $t_{0}$, and $\langle\cdot\rangle$ is the ensemble average. Vibrational density of states (VDOS) of the phonon with frequency $v$ can be obtained:

$$
\operatorname{VDOS}(\nu)=\int_{-\infty}^{+\infty} \operatorname{VACF}(t) e^{-2 \pi i v t} \mathrm{~d} t .
$$

Periodic boundary conditions were used in this simulation. First, the energy of the system was minimized. In order to ensure the stability of the calculation system, the time step was $0.2 \mathrm{fs}$. Then, NPT ensemble and NVT ensemble were implemented, respectively, and the structure was optimized at constant temperature for $0.4 \mathrm{~ns}$. Then, the system was switched to constant energy (NVE) simulations for 20 ns. During this period, the temperature distribution curve (as shown in Figure 3) was obtained once per $1 \mathrm{~ns}$, and the average temperature difference was output. According to the formula [2], the thermal conductivity and VACF can be calculated by using the data after $1 \mathrm{~ns}$.

The simulated thermal conductivity of single-layer g- $\mathrm{C}_{3} \mathrm{~N}_{4}(14.76 \times 12.78 \times 0.5 \mathrm{~nm})$ is about $2.93 \mathrm{~W} /(\mathrm{K} \bullet \mathrm{m})$, which is comparable to the result calculated by equilibrium $\mathrm{MD}$ based on the Green-Kubo formula $(3.5 \pm 0.3 \mathrm{~W} / \mathrm{K} \bullet \mathrm{m})$ [15]. This proves the reliability of the methodology adopted here in describing the thermal properties of $g-\mathrm{C}_{3} \mathrm{~N}_{4}$ systems.

\section{Results and Discussion}

3.1. Stability of the Nanotubes. The maximum diameter of the nanotubes $(2.5 \mathrm{~nm})$ studied here is limited mainly by the computation cost, which results in a strong internal stress. It is then easy to cause nanotube collapse and deformation with increasing temperature, leading to a meaningless calculation. However, nanotubes with different diameters can approximately retain their structure if the temperature is lower than $600 \mathrm{~K}$, as shown in Figure 4. Although there still exists tiny deformation, the simulated result of the thermal conductivity is quite reliable.

The corresponding relation between the $(m, n)$ vector and diameters can be referred to Table 1 , and it is clear in Figure 4 that the basic orientation of the tube can significantly affect the shape of the tube. When the diameter is small, the tube shows a distorted shape. When the diameter increases, the tube wall becomes smoother, and the tube becomes straighter. And when the value of $m$ and $n$ is small, the atom distribution is generally sparse. The structure of the tubes may further influence the thermal properties of the tubes, which will be discussed later.

3.2. Size Effects on the Thermal Conductivity of the Nanotube. Surface stress exists while rolling the two-dimensional g- $\mathrm{C}_{3} \mathrm{~N}_{4}$ layers along different chiral angles. The stress is the 


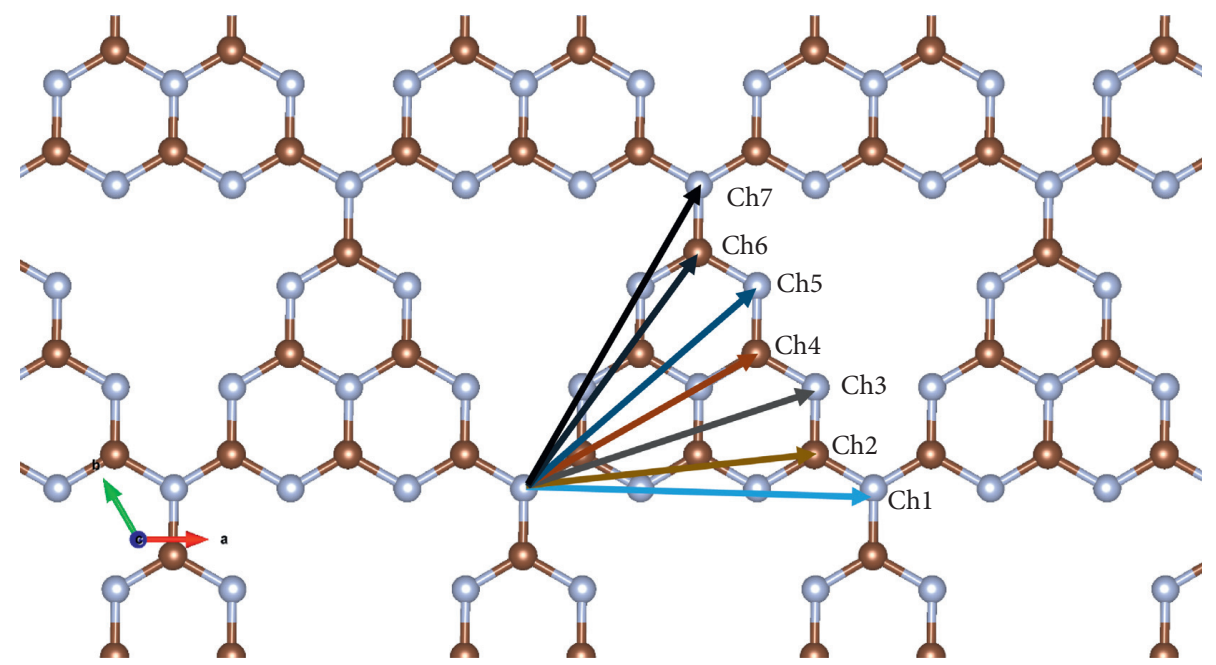

Figure 1: Nanotube cells defined by the chiral vector $\mathrm{Ch}=(n, m)$, where $n$ and $m$ are vector components in two different directions.

TABLE 1: The information of the constructed nanotubes and the computational parameters.

\begin{tabular}{lccccc}
\hline Chiral vector & $(n, m)$ & Chiral angle $\left(^{\circ}\right)$ & Diameter $(\mathrm{nm})$ & Length of the nanotube $(\mathrm{nm})$ & Number of atoms \\
\hline Ch1 & $(3,0)$ & 0 & 0.71 & 11.50 & 11.50 \\
Ch1 & $(5,0)$ & 0 & 1.18 & 11.50 & 1260 \\
Ch1 & $(7,0)$ & 0 & 1.72 & 11.50 & 1764 \\
Ch1 & $(9,0)$ & 0 & 2.13 & 11.50 & 2268 \\
Ch1 & $(10,0)$ & 0 & 2.35 & 11.50 & 2520 \\
Ch1 & $(11,0)$ & 0 & 2.59 & 12.19 & 2772 \\
Ch2 & $(10,1)$ & 6 & 2.24 & 13.53 & 2548 \\
Ch3 & $(12,4)$ & 20 & 2.49 & 11.81 & 3136 \\
Ch4 & $(10,5)$ & 30 & 2.05 & 12.55 & 2240 \\
Ch5 & $(12,8)$ & 40 & 2.09 & 12.19 & 2380 \\
Ch6 & $(10,9)$ & 54 & 2.27 & 11.50 & 2548 \\
Ch7 & $(10,10)$ & 60 & 2.35 & & 2520 \\
\hline
\end{tabular}

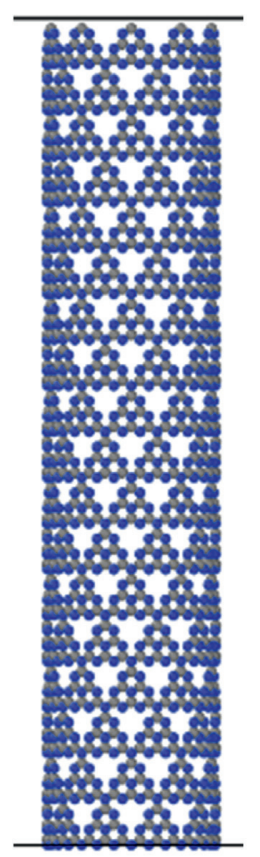

$(10,0) 0^{\circ}$

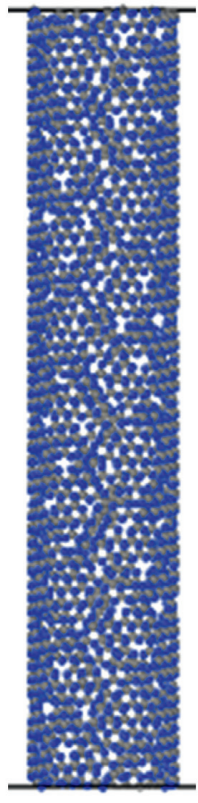

$(10,1) 6^{\circ}$

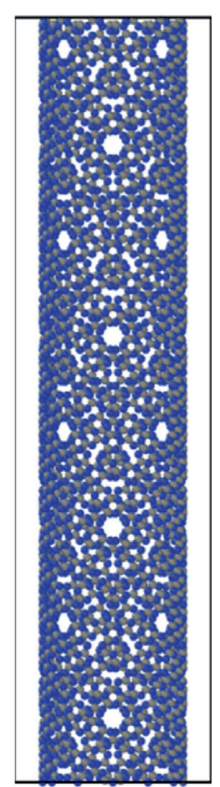

$(12,4) 20^{\circ}$

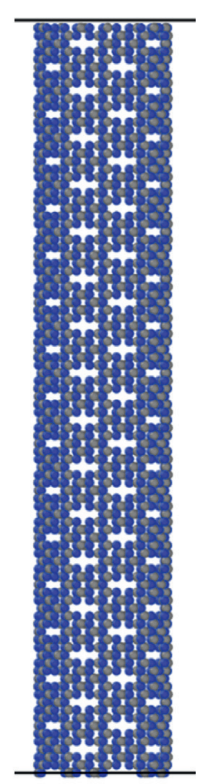

$(10,5) 30^{\circ}$

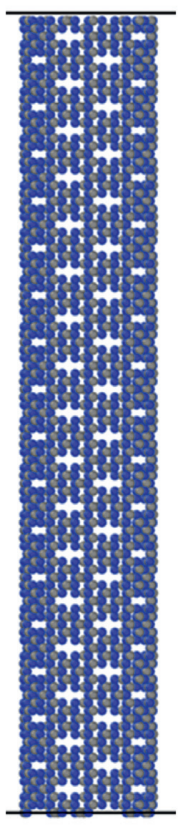

$(12,8) 40^{\circ}$

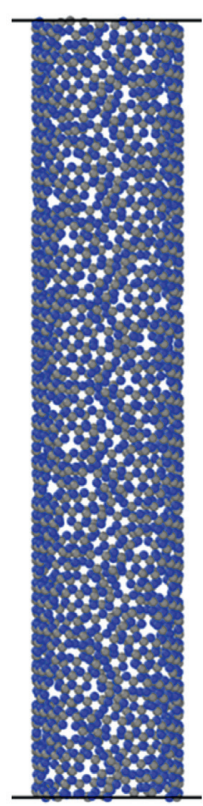

$(10,9) 54^{\circ}$

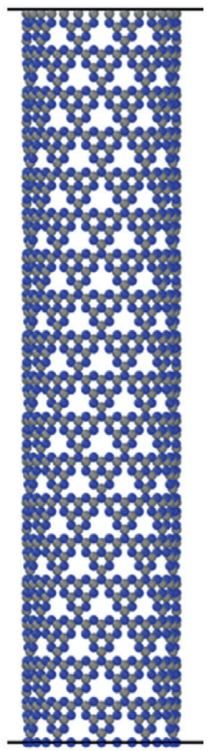

$(10,10) 60^{\circ}$

FIgURe 2: Side view of nanotubes at different chiral angles. 


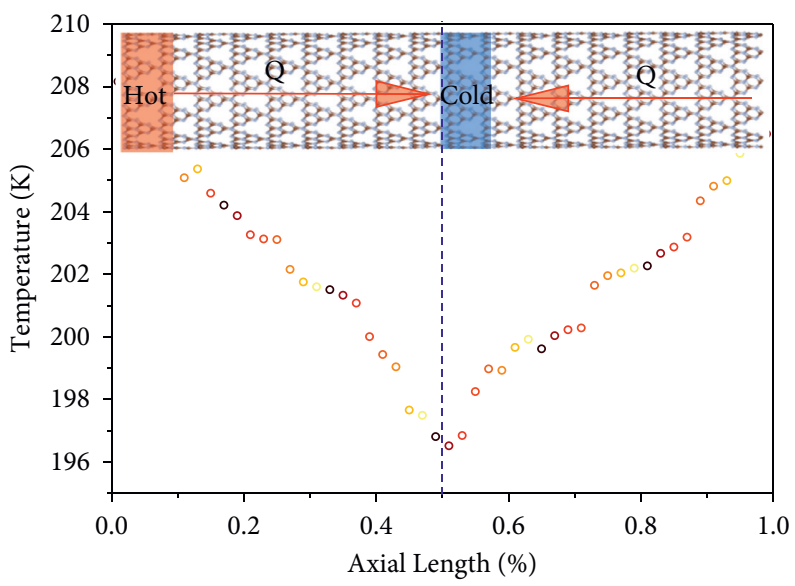

Figure 3: Schematic diagram of the thermal conductivity calculation of g- $\mathrm{C}_{3} \mathrm{~N}_{4}$.

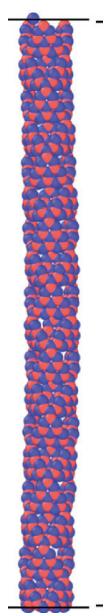

$(3,0)$

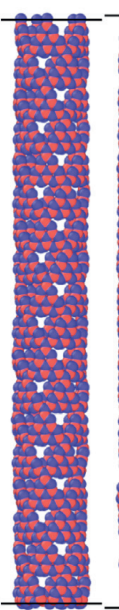

$(5,0)$

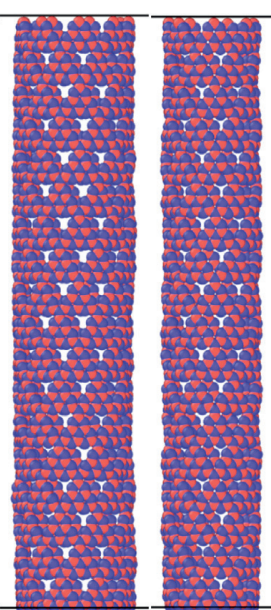

$(7,0)$

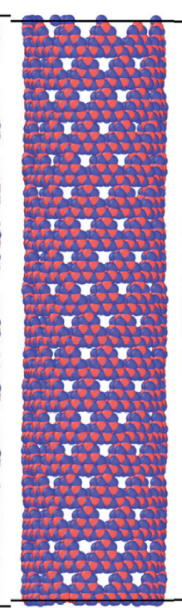

$(11,0)$

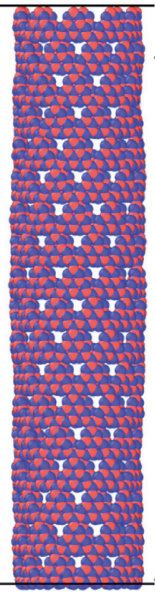

$(10,0)$

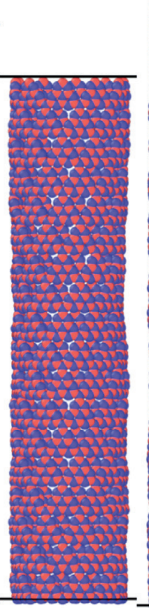

$(10,1)$

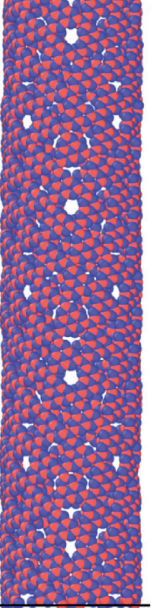

$(12,4)$

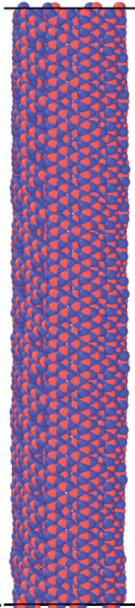

$(10,5)$

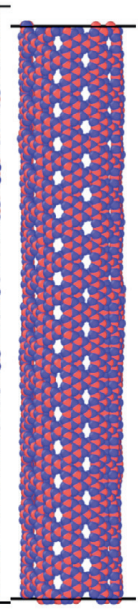

$(12,8)$

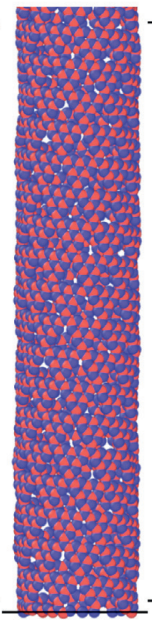

$(10,9)$

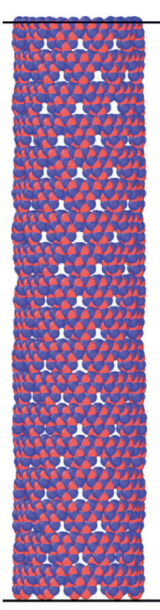

$(10,10)$

FIGURE 4: Nanotubes with different diameters at temperature less than $600 \mathrm{~K}$.

change of phonon density of states caused by the atomic force in the microscopic scale, while the macroscopic performance is quite different. For nanotubes with different diameters, the number of atoms in the same plane is different. To figure out the intrinsic size effects of the thermal conductivity of nanotubes, the thermal conductivity per unit arc length is introduced [16]:

$$
\kappa_{s}=\frac{\kappa}{\pi d},
$$

where $d$ is the nanotube diameter.

The thermal conductivity per unit arc length of g- $\mathrm{C}_{3} \mathrm{~N}_{4}$ with outer diameter from 3 to $17 \mathrm{~nm}$ varies little with temperature in the range of $200-600 \mathrm{~K}$ (as shown in Figure 5), which is very different from that of other carbon nanotubes [16].

As shown in Figure 6, the thermal conductivity per unit arc length of nanotubes decreases significantly with increasing diameters. This indicates that the internal tension between the atoms of nanotubes becomes weaker with larger diameter, which makes it difficult for the thermal vibration

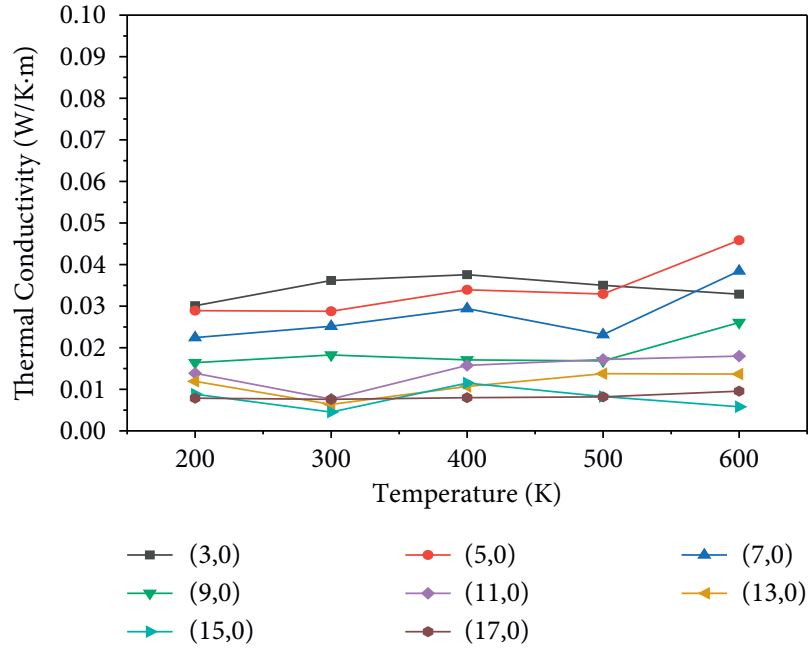

FIgURE 5: The temperature dependence of the thermal conductivity of unit arc length of $\mathrm{g}-\mathrm{C}_{3} \mathrm{~N}_{4}$ with outer diameters from 3 to $17 \mathrm{~nm}$. 


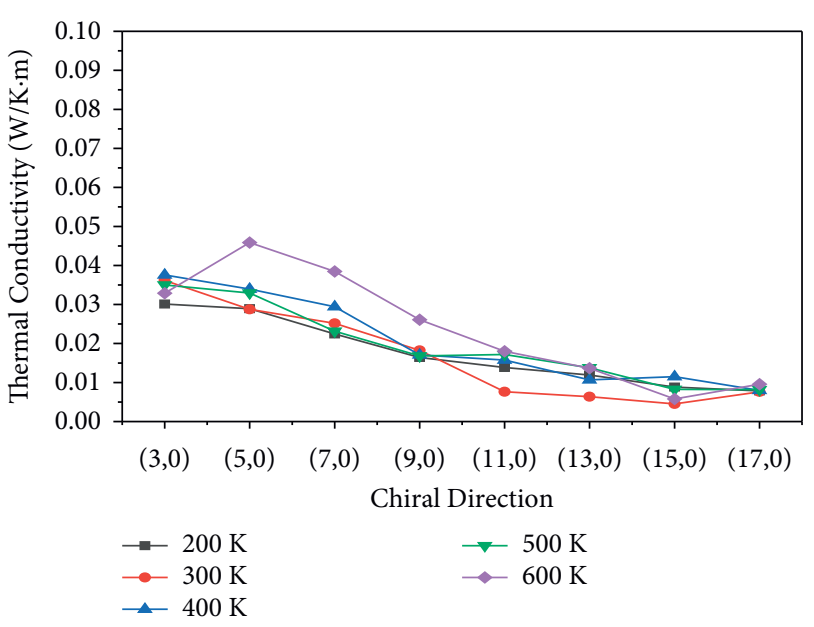

Figure 6: The diameter dependence of the thermal conductivity of unit arc length of $\mathrm{g}-\mathrm{C}_{3} \mathrm{~N}_{4}$ nanotubes.

between adjacent atoms to propagate smoothly along the axial direction.

3.3. Chirality Effects on the Thermal Conductivity of the Nanotube. Rolling two-dimensional materials along different chiral angles can form nanotube structures with different chiral angles, leading to different atomic arrangements with different physicochemical properties. Therefore, it is meaningful to study the influence of chiral angles on the thermal conductivity of nanotubes. As shown in Figure 7, the thermal conductivity of a single chiral nanotube varies little with the temperature in the range of 200-600 K. For nanotubes with different chiral angles, the atomic thermal vibration of the nanotubes is intensified, but the contribution to heat conduction is limited.

The thermal conductivity of nanotubes varies little with the chiral angles under the same temperature, as shown in Figure 8, which indicates that it is impossible to adjust the thermal conductivity by changing only the chiral angles of nanotubes. We have shown in Figure 4 that the chiral angles can influence both diameter and atom distribution of the nanotubes. Generally speaking, a dense atom distribution on the tube walls is apt to increase the thermal conductivity. On the contrary, smaller diameter will increase the atom density in the $3 \mathrm{D}$ space, which is likely to increase the thermal conductivity. The two factors are contradictory. Thus, when $m$ and $n$ are small, the atom distribution on the tube wall is sparse, yet the diameter is small, compensating the decrease of thermal conductivity. Thus, the final thermal conductivity may be decided by the relation of the two factors.

3.4. Vibrational Density of States (VDOS). In order to gain more insight into the chirality effects on the thermal conductivity of nanotubes, the VDOS of nanotubes was calculated since vibrational properties at low frequency are corresponding to the thermal conductivity property. As shown in Figure 9, the VDOS of nanotubes at different chiral angles calculated at low temperature $(300 \mathrm{~K})$ was mainly

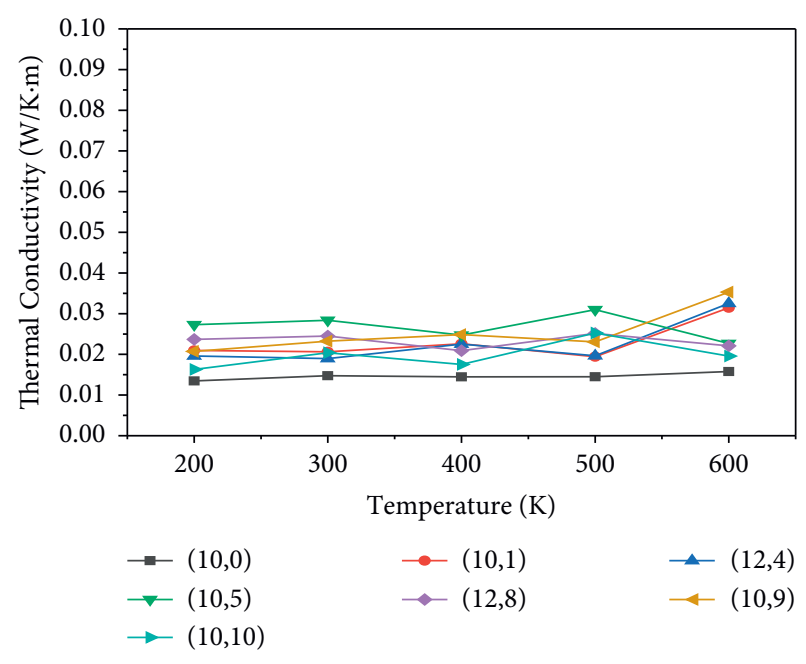

FIgURE 7: The temperature dependence of the thermal conductivity of different chiral nanotubes.

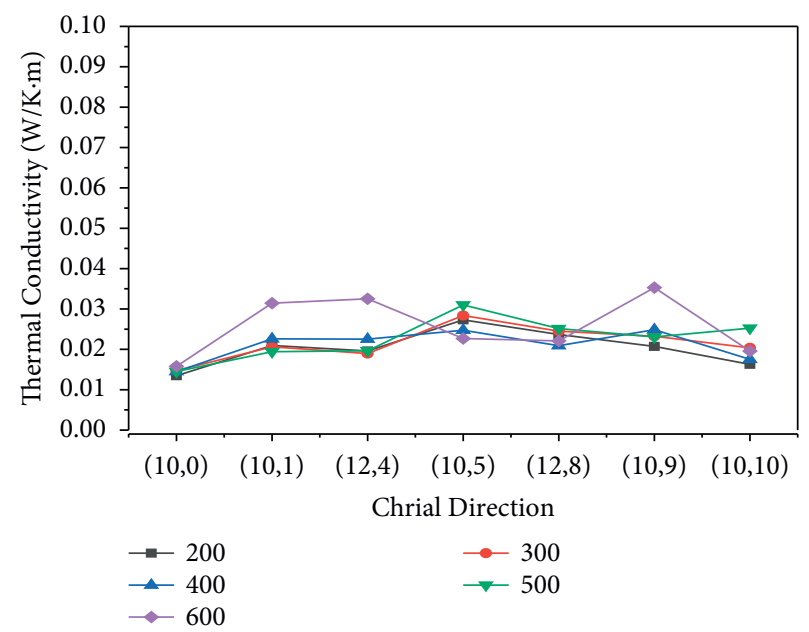

FIgURE 8: The chiral angles' dependence of the thermal conductivity at single temperature.

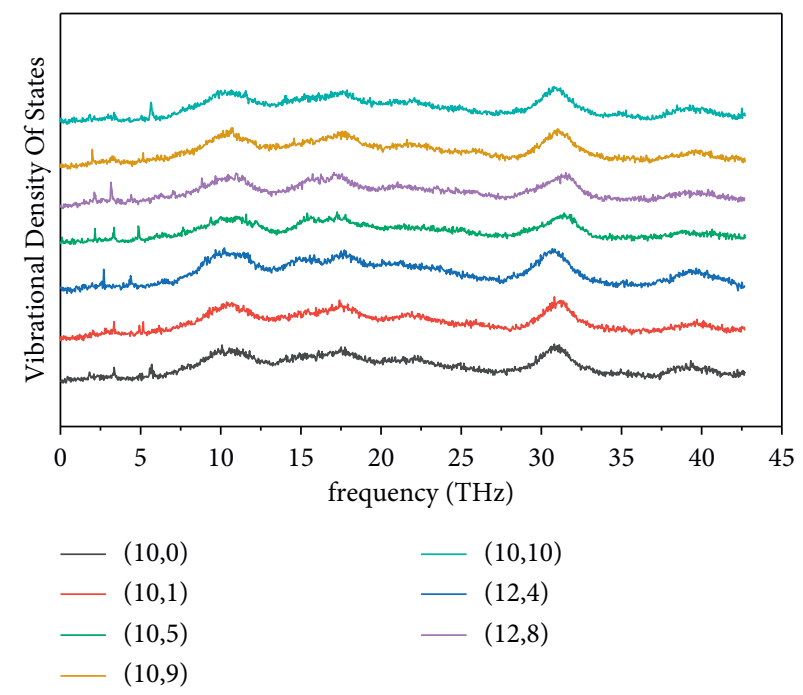

FIGURE 9: Vibrational density of states (VODS) of nanotubes with different chiral angles at temperature $300 \mathrm{~K}$. 
concentrated in frequencies $\sim 11 \mathrm{THz}$ and $\sim 32 \mathrm{THz}$. There are few vibrational modes at frequencies lower than $10 \mathrm{THz}$, which respond mainly to effective heat conduction. Therefore, the thermal conductivity of nanotubes with different chiral angles was very low. This is also true for the VDOS at different temperatures with the same diameter or chiral angle. In general, it can be seen that the vibrational modes of $\mathrm{g}-\mathrm{C}_{3} \mathrm{~N}_{4}$ at low frequency are quite few, and heating or changing chiral angles plays a minor role in adjusting the thermal conductivity.

\section{Conclusions}

In summary, the dependence of the thermal conductivity of g- $\mathrm{C}_{3} \mathrm{~N}_{4}$ nanotubes on temperature, diameters, and chiral angles of the nanotubes was characterized by molecular dynamics study. The simulation results suggest that, for a single nanotube, the thermal conductivity is not sensitive to temperature and chiral angles, while under the same temperature, the thermal conductivity decreases significantly with increasing nanotube diameters. These results provide useful thermal information in guiding the design and application of $\mathrm{g}-\mathrm{C}_{3} \mathrm{~N}_{4}$ nanotubes in the future devices.

\section{Data Availability}

The data used to support the findings of this study are included within the article.

\section{Conflicts of Interest}

The authors declare no conflicts of interest.

\section{Acknowledgments}

This work was supported by the Hainan Provincial Natural Science Foundation of China (Grant no. 519MS025) and the Education and Teaching Research Project Funding of Hainan University (Grant no. hdjy2160).

\section{References}

[1] J. Gao, Y. Zhou, Z. Li, S. Yan, N. Wang, and Z. Zou, "Highyield synthesis of millimetre-long, semiconducting carbon nitride nanotubes with intense photoluminescence emission and reproducible photoconductivity," Nanoscale, vol. 4, no. 12, pp. 3687-3692, 2012.

[2] C. Han, Y. Wang, Y. Lei et al., "In situ synthesis of graphitic$\mathrm{C}_{3} \mathrm{~N}_{4}$ nanosheet hybridized $\mathrm{N}$-doped $\mathrm{TiO} 2$ nanofibers for efficient photocatalytic $\mathrm{H}_{2}$ production and degradation," Nano Research, vol. 8, no. 4, pp. 1199-1209, 2015.

[3] Y. Zheng, J. Liu, J. Liang, M. Jaroniec, and S. Z. Qiao, "Graphitic carbon nitride materials: controllable synthesis and applications in fuel cells and photocatalysis," Energy \& Environmental Science, vol. 5, no. 5, pp. 6717-6731, 2012.

[4] T. N. Yan, E. H. Liu, Z. Y. Chu, and X. D. Li, "Scalable synthesis of ultrathin $\mathrm{g}-\mathrm{C}_{3} \mathrm{~N}_{4}$ nanosheets with enhanced photocatalytic activities via high shear exfoliation," Journal of Nano Research, vol. 45, pp. 49-54, 2017.

[5] S. Sun, E. Fan, H. Xu et al., "Enhancement of photocatalytic activity of $\mathrm{g}^{-} \mathrm{C}_{3} \mathrm{~N}_{4}$ by hydrochloric acid treatment of melamine," Nanotechnology, vol. 30, no. 31, Article ID 315601, 2019.

[6] F. K. Kessler, Y. Zheng, D. Schwarz et al., "Functional carbon nitride materials-design strategies for electrochemical devices," Nature Reviews Materials, vol. 2, no. 6, pp. 1-17, 2017.

[7] X. Li, K. Pan, Y. Qu, and G. Wang, "One-dimension carbon self-doping $\mathrm{g}-\mathrm{C}_{3} \mathrm{~N}_{4}$ nanotubes: synthesis and application in dye-sensitized solar cells," Nano Research, vol. 11, no. 3, pp. 1322-1330, 2018.

[8] S.-W. Bian, Z. Ma, and W.-G. Song, "Preparation and characterization of carbon nitride nanotubes and their applications as catalyst supporter," Journal of Physical Chemistry C, vol. 113, no. 20, pp. 8668-8672, 2009.

[9] Z. Jin, Q. Zhang, S. Yuan, and T. Ohno, "Synthesis high specific surface area nanotube g- $\mathrm{C}_{3} \mathrm{~N}_{4}$ with two-step condensation treatment of melamine to enhance photocatalysis properties," RSC Advances, vol. 5, no. 6, pp. 4026-4029, 2015.

[10] Y. Miyamoto, M. L. Cohen, and S. G. Louie, "Theoretical investigation of graphitic carbon nitride and possible tubule forms," Solid State Communications, vol. 102, no. 8, pp. 605-608, 1997.

[11] H. Pan, Y. Zhang, V. B. Shenoy, and H. Gao, "Ab initio study on a novel photocatalyst: functionalized graphitic carbon nitride nanotube," ACS Catalysis, vol. 1, no. 1, pp. 99-104, 2011.

[12] K. Momma and F. Izumi, "VESTA 3 for three-dimensional visualization of crystal, volumetric and morphology data," Journal of Applied Crystallography, vol. 44, no. 6, pp. 1272-1276, 2011.

[13] S. Plimpton, "Fast parallel algorithms for short-range molecular dynamics," Journal of Computational Physics, vol. 117, no. 1, pp. 1-19, 1995.

[14] A. Kinaci, J. B. Haskins, C. Sevik, and T. Cagin, "Thermal conductivity of BN-C nanostructures," Physical Review B, vol. 86, no. 11, pp. 1-8, 2012.

[15] B. Mortazavi, G. Cuniberti, and T. Rabczuk, "Mechanical properties and thermal conductivity of graphitic carbon nitride: a molecular dynamics study," Computational Materials Science, vol. 99, pp. 285-289, 2015.

[16] L. Yang, C.-Z. Wang, S. Lin et al., "Thermal conductivity of $\mathrm{TiO}_{2}$ nanotube: a molecular dynamics study," Journal of Physics: Condensed Matter, vol. 31, no. 5, Article ID 055302, 2019. 